\title{
Study on a Concentration Sensor with a Hot Wire to Measure the Composition of a Binary Gas Mixture*
}

\author{
Motoaki KIMURA** and Norimasa MIYAGI*** \\ **Department of Mechanical Engineering, College of Science \& Technology, Nihon University, \\ 1-8-14 Kanda-Surugadai, Chiyoda-ku, Tokyo 101-8308, Japan \\ E-mail: kimura@mech.cst.nihon-u.ac.jp \\ ***Junior College Funabashi Campus, Nihon University, \\ 7-24-1 Narashinodai, Funabashi, Chiba 274-8501, Japan
}

\begin{abstract}
The present experimental study is an attempt to measure the concentration of jet diffusion. A hot-wire concentration sensor is used to measure the concentration of the components in a binary mixture of gases. King's equation, which gives the heat transfer from a fine wire heated by Joule heating in a gas flow with a low Reynolds number, includes two main parameters, velocity and thermal conductivity. The effect of velocity is suppressed by the passage of gases through a sonic nozzle. Thus, in the hot wire, the influence of thermal conductivity exceeds that of velocity. The concentration probe is calibrated using a helium jet and a carbon dioxide jet. The calibration curve is prepared using a helium-air mixture and a carbon dioxide-air mixture. The time constant of the probe is measured to be $0.74 \mathrm{~ms}$ for helium and $3.48 \mathrm{~ms}$ for carbon dioxide. The contours of velocity and concentration of helium and carbon dioxide gases are measured.
\end{abstract}

Key words: Jet, Diffusion, Concentration Sensor, Hot-Wire, Concentration Distribution

\section{Introduction}

Gas mixture by jet flow diffusion is important in relation to combustion enhancement, stability control, and combustion products. Control of jet flow diffusion has been attempted by inducing an initial disturbance by means of the nozzle exit shape ${ }^{(1)}$ and the preferred frequency of the jet flow ${ }^{(2)}$. As for the characteristics of the jet flow diffusion, investigation of the average velocity, the turbulence velocity, and the frequency analysis, for example, has been actively performed by hot-wire anemometer, LDV, and PIV, for example (3). In diffusion combustion, the combustion of the gas in a mixture advances in the place where the gas in a mixture of the fuel and air reached within the range of combustion. Therefore, both momentum transportation control and material transportation control are necessary for controlling the combustion conditions. Kurimoto et al. investigated the stability improvement of combustion by installing a MEMS actuator in the nozzle and applying an initial disturbance ${ }^{(4)}$. They evaluated the concentration diffusion from the probability density distribution of the concentration based on visualization data. A high-response concentration sensor is needed to measure the mass diffusion of the free shear layer of a jet flow. Gas sensors have been used for a variety of applications, including disaster prevention, industrial fields, air pollution detection, and food fields. Semiconductor gas sensors have

*Received 15 Dec., 2010 (№. 10-0603) [DOI: 10.1299/jfst.6.674]

Copyright $\odot 2011$ by JSME 
primarily been used for gas detection. There are two types of semiconductor gas sensors, namely, the electrochemistry type and the solid electrolysis type, which makes use of the phenomenon whereby the electric conductivity of a metallic oxide changes due to contact with the gas ${ }^{(5)}$. In addition, the gas chromatograph and the mass spectrometer have been used for gas analysis. The response times of these devices range from 0.1 to $1 \mathrm{~s}$. A response time on the order of several milliseconds may be necessary in order to measure the change in concentration of jet flow diffusion. Brown and Rebollo ${ }^{(6)}$ developed a probe with which to measure the composition of a binary gas mixture using a hot-wire. Era ${ }^{(7)}$ and Muramatsu ${ }^{(8)}$ improved the probe sensitivity and response by carefully manufacturing the probe tube holding the hot-wire prong from quartz.

The concentration sensor considered herein is composed of a sonic nozzle constructed of brass and a hot-wire anemometer. A minute nozzle hole is drilled using an Nd:YAG pulse laser. The concentration of binary mixture gases is detected from the change in thermal conductivity of the gas mixture. The static and dynamic characteristics of the concentration sensor with a hot wire are described in $\S 3$. Example measurements of the diffusion characteristics of the helium jet and the carbon dioxide jet measured using the sensor are described in $\S 4$.

\section{Nomenclature}

$\begin{array}{ll}d: & \text { nozzle diameter } \\ d_{w}: & \text { diameter of hot wire } \\ E_{\mathrm{air}}: & \text { sensor output for air } \\ E_{\mathrm{CO} 2}: & \text { sensor output for pure } \mathrm{CO}_{2} \\ E_{\mathrm{He}}: & \text { sensor output for pure } \mathrm{He} \\ E_{\mathrm{mix}}: & \text { sensor output for gas mixture } \\ E_{f}: & \text { sensor output for the full range }\left(E_{\mathrm{He}}-E_{\mathrm{air}}, E_{\mathrm{CO} 2}-E_{\mathrm{air}}\right) \\ E_{s}: & \text { sensor output }\left(E_{\mathrm{mix}}-E_{\mathrm{air}}\right) \\ I: & \text { bridge current } \\ l_{w}: & \text { length of hot wire } \\ P_{i n}: & \text { probe inner pressure } \\ P_{o}: & \text { atmospheric pressure } \\ P(U): & \text { probability density function of velocity } \\ P(\Lambda): & \text { probability density function of concentration } \\ R e: & \text { Reynolds number } \\ r: & \text { radial coordinate } \\ S: & \text { gap between the sonic nozzle exit and the hot wire } \\ T_{o}: & \text { atmospheric temperature } \\ U: & \text { flow velocity } \\ U_{o}: & \text { flow velocity at the nozzle exit } \\ u: & \text { flow velocity at the hot wire } \\ X: & \text { streamwise coordinate } \\ \kappa: & \text { specific heat ratio of gases } \\ \Lambda: & \text { volume concentration } \\ \Lambda: & \text { fluctuating volume concentration (r.m.s. }) \\ \tau: & \text { time constant }\end{array}$

\section{Experimental Apparatus}

\subsection{Principles}

Figure 1 shows the arrangement of the sonic nozzle (diameter: $d_{t}$ ) at the tip of the probe of the concentration sensor and the hot wire. The gas is accelerated to the speed of sound by 
the sonic nozzle by reducing the pressure in the probe to below the critical pressure, and the gas reaches the tungsten hot wire. The concentration of a binary gas mixture can be measured because the change in heat discharge from the Joule heated hot wire is related to the velocity and the thermal conductivity of the gas. In general, in King's equation, the heat discharge from the heated hot wire depends on the square root of the velocity and the thermal conductivity of the gas to be measured:

$$
\begin{gathered}
I^{2} R_{W}=A I_{W} \lambda+B I_{W} \sqrt{C_{p} \lambda \rho d_{W} u} \\
A=\frac{a\left(R_{W}-R_{g}\right)}{R_{20} \alpha} \quad B=\frac{b\left(R_{W}-R_{g}\right)}{R_{20} \alpha}
\end{gathered}
$$

where $I$ is the bridge current, $R_{w}$ is the resistance of the hot wire, $R_{\mathrm{g}}$ is the resistance of the hot wire at the flow temperature, $R_{20}$ is the resistance of the hot-wire at $20^{\circ} \mathrm{C}, \alpha$ is the temperature coefficient of resistivity, $a$ and $b$ are calibration constants, $l_{w}$ is the length of the hot wire, $d_{w}$ is the diameter of the hot wire, $\lambda$ is the thermal conductivity of gas, $C_{p}$ is the specific heat at constant pressure of the gas, $\rho$ is the density of the gas, and $u$ is the flow velocity at the hot-wire. Since the thermal conductivity in a binary gas mixture changes depending on the concentration, the velocity and concentration influence the heat discharge of the hot wire. The measured gas from the sonic nozzle is sucked into the probe at the speed of sound and expanded to make the velocity uniform, thereby minimizing the influence of the initial velocity distribution ${ }^{(6)}$. The concentration is measured from the thermal conductivity of the gas by arranging the hot wire at the location where the velocity becomes constant.

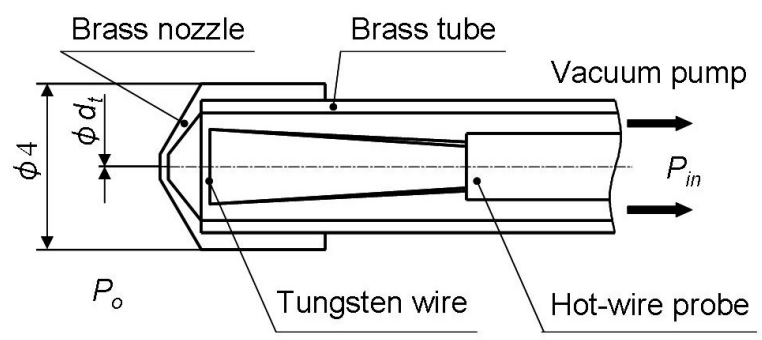

Fig. 1 Nozzle and hot-wire of the concentration sensor

\subsection{Concentration Sensor System}

Figure 2 shows the setup of the hot-wire concentration sensor. The sensor consists of a concentration probe, a vacuum pump, a pressure adjustment needle valve, a vacuum gauge, and a hot-wire anemometer. Figure 1 shows the tip of the concentration probe. The concentration probe consisted of a brass sonic nozzle, a brass tube, and a hot wire. The sonic nozzle was drilled using an Nd:YAG pulse laser. Several nozzle diameters $d_{t}$ ranging from 30 to $140 \mu \mathrm{m}$ were prepared. The tungsten hot-wire had a diameter of $4 \mu \mathrm{m}$ and a length of $1.8 \mathrm{~mm}$. The pressure inside the brass probe was reduced using the vacuum pump and the pressure adjustment needle valve. The inside-to-outside pressure ratio of the probe $P_{i n} / P_{o}$ was varied from 0.02 to 0.1 . The velocity of the gas at the sonic nozzle reached the speed of sound, and the accelerated gas reaches the hot wire. The output of the hot wire is connected to the anemometer. The static and dynamic characteristics of the sensor are investigated as described in the following chapter. 


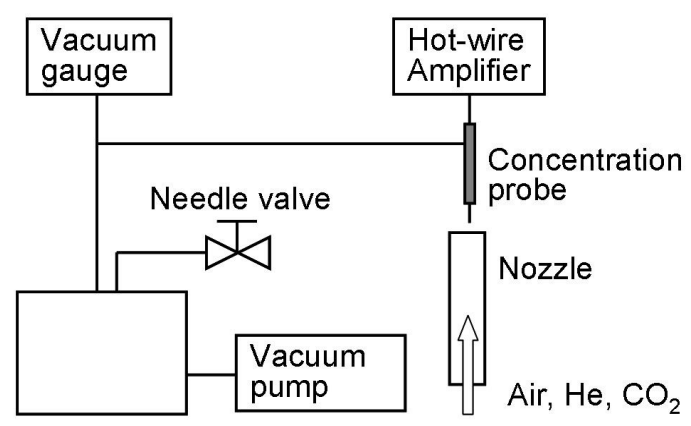

Fig. 2 Concentration sensor system

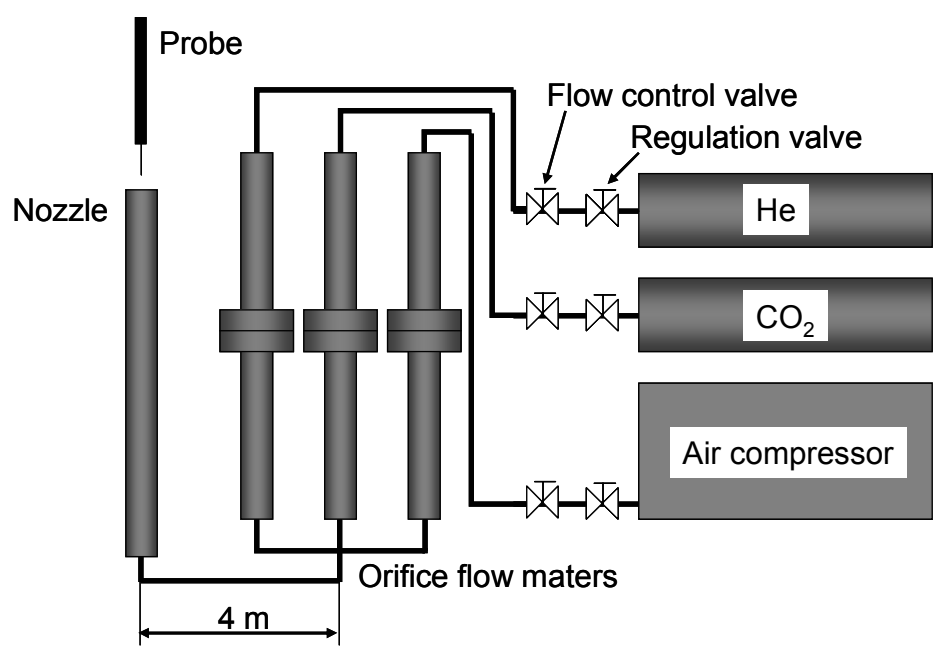

Fig. 3 Gas mixing device

\section{Sensitivity and Response of the Hot-wire Concentration Sensor}

\subsection{Sensitivity}

Figure 3 shows the device used for the calibration of the sensitivity of the sensor. High-pressure gas cylinders and the compressor supplied helium, carbon dioxide, and air through the regulation valve to maintain the pressure as constant. A binary gas mixture was prepared by mixing air with a pure gas, as measured by the orifice flow meters and the flow control valves. The measured pure gas and air were mixed by passing through a $4 \mathrm{~m}$ tube. The mixture gas was issued from the nozzle of a tube of $10 \mathrm{~mm}$ in diameter. The sensor probe tip was calibrated by placing the tip in the concentration potential core area of a jet flow in which the concentration was constant. The device was able to regulate the gas volume concentration as $\Lambda=0$ to $100 \%$ :

$$
\begin{array}{ll}
\Lambda=\frac{V_{H e}}{V_{A i r}+V_{H e}} & \Lambda=\frac{V_{C O 2}}{V_{A i r}+V_{C O 2}} \\
\frac{E_{S}}{E_{f}}=\frac{E_{\text {mix }}-E_{\text {air }}}{E_{C O 2}-E_{\text {air }}} & \frac{E_{S}}{E_{f}}=\frac{E_{\text {mix }}-E_{\text {air }}}{E_{H e}-E_{\text {air }}}
\end{array}
$$

where $V_{\text {air }}, V_{\mathrm{CO} 2}$, and $V_{\mathrm{He}}$ are the volume flow rates of air, $\mathrm{CO}_{2}$, and $\mathrm{He}$, respectively.

The relationship between the volume concentration and the sensor output is shown. Figures 4 and 5 show the relationship between the measured volume concentration $\Lambda$ and the sensor output $E_{s} / E_{f}$, where $E_{s}$ is the sensor output for mixture gas and $E_{f}$ is the sensor 
output for the full range. The experimental conditions were as follows. The pressure ratio of the probe $P_{i n} / P_{o}$ was 0.02 . The gap between the sonic nozzle exit and the hot wire $S$ was $0.65 \mathrm{~mm}$, and the sonic nozzle diameter $d_{t}$ was $55 \mu \mathrm{m}$. The external flow velocity $U_{o}$ was 20 $\mathrm{m} / \mathrm{s}$ for the carbon dioxide-air mixture and $50 \mathrm{~m} / \mathrm{s}$ for the helium-air mixture. The solid lines in the figures indicate the value obtained from King's equation using the thermal conductivity of the binary mixture gas. In Fig. 4, the relationship between the volume concentration and the sensor output is approximately proportional to the binary gas mixture of carbon dioxide and air. On the other hand, this is not the case for the binary gas mixture of helium and air, as shown in Fig. 5. This is thought to be due to the fact that the change in the concentration with the thermal conductivity of the binary gas mixture of helium and air, which is a predominant variable of the sensor, is not linear.

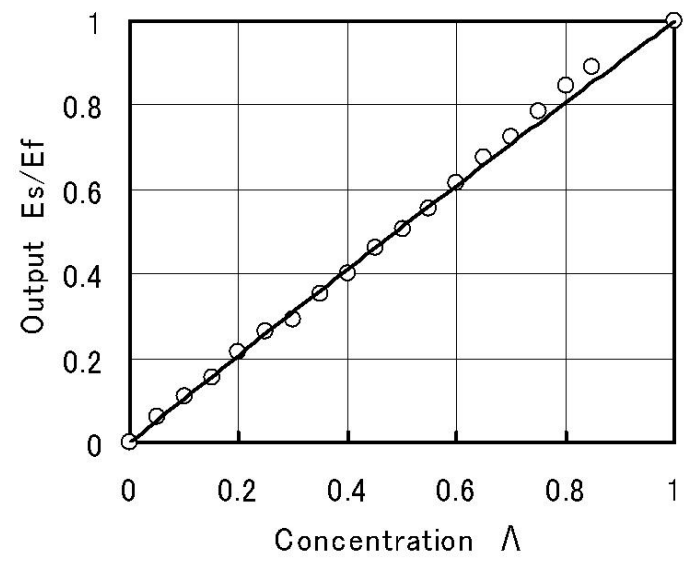

Fig. 4 Sensitivity in the gas mixture of $\mathrm{CO}_{2}$ and air

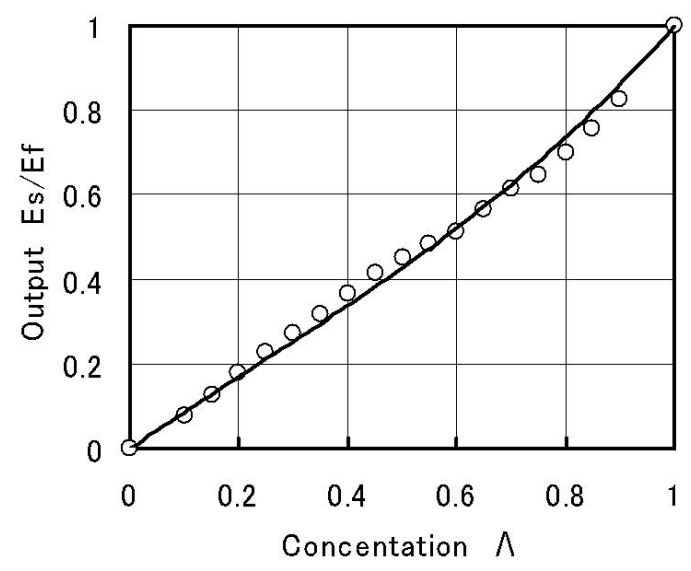

Fig. 5 Sensitivity in the gas mixture of $\mathrm{He}$ and air

\subsection{Dynamic Characteristics}

The dynamic characteristics were measured using the rotary dynamic characteristic measuring device shown in Fig. 6. The device consists of a swing arm with a radius of 250 $\mathrm{mm}$, a sensor holder, and a driving motor. The sensor was rotated by the arm and the powered motor. A nozzle with a diameter of $10 \mathrm{~mm}$ was arranged at the position over which the sensor tip passed. The gap between the nozzle exit and the tip of the concentration probe was set to be in the range from 0.5 to $1 \mathrm{~mm}$. Pure helium or pure carbon dioxide $(\Lambda=$ $100 \%$ ) was vertically issued from the tube nozzle. A step change in concentration was caused when the probe crossed the jet flow from the tube. The time constant of the hot-wire concentration sensor was measured, and the dynamic characteristics were evaluated. The measurement parameters were the external flow velocity, $U_{o}$, the volume concentration, $\Lambda$, 
the gap between the sonic nozzle exit and the hot wire, $S$, the pressure ratio of the probe, $P_{i n} / P_{o}$, and the sonic nozzle diameter, $d_{t}$.

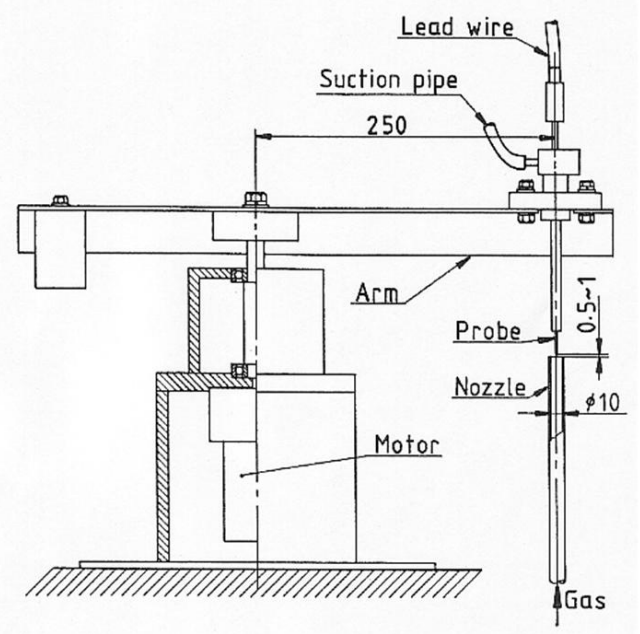

Fig. 6 Calibration apparatus for dynamic sensitivity (units: $\mathrm{mm}$ )

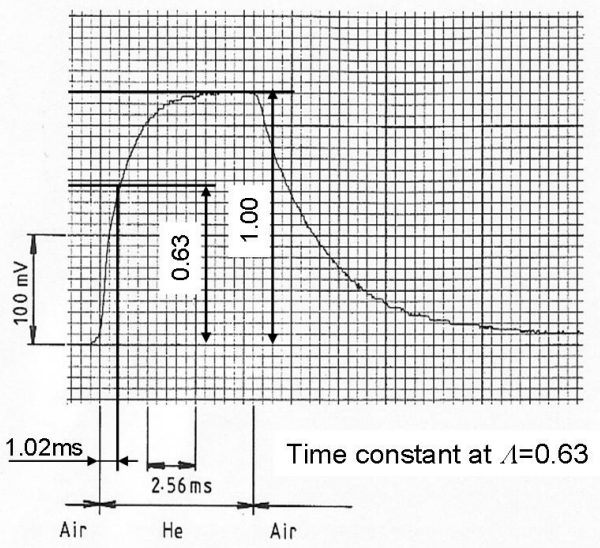

Fig. 7 Output signal of He with air

Figure 7 shows an example of the wave shape of the dynamic response characteristics of the sensor obtained by this method. The measurement condition was as follows: external flow velocity; $20 \mathrm{~m} / \mathrm{s}$, issuing gas; helium, pressure ratio; 0.02, and sonic nozzle exit diameter; $55 \mu \mathrm{m}$. The dynamic characteristics were evaluated by defining the time constant: $\tau$, as the time at which the volume concentration reached 0.63 from 0 . The relationship between $P_{i n} / P_{o}$ and $\tau$ for sonic nozzles of various exit diameters is shown in Fig. 8. The time constant decreases as $P_{i n} / P_{o}$ decreases. In addition, when the space inside the concentration probe is decompressed, the time constant exhibits a tendency to approach a finite value. The time constant decreases with the increase in the diameter of the sonic nozzle, as shown in Fig. 8. However, when the suction flow rate is increased, it is preferable not to increase the diameter of the sonic nozzle because of the disturbance introduced to the measured flow field. The time constants are $\tau=0.74 \mathrm{~ms}$ for helium and $\tau=3.48 \mathrm{~ms}$ for carbon dioxide for a gap between the sonic nozzle exit and the hot wire of $0.65 \mathrm{~mm}, P_{i n} / P_{o}=0.02$, and a sonic nozzle diameter of $55 \mu \mathrm{m}$. 


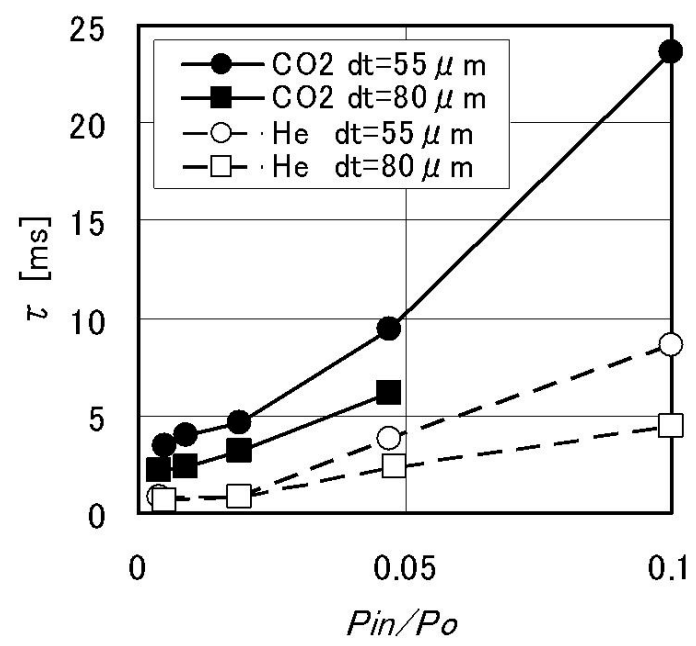

Fig. 8 Relationship between pressure ratio and dynamic sensitivity

The suction flow rate calculated for the sonic nozzle is shown for the case of a compressible flow. For a choked flow, the Mach number $M a=1$, and the pressure, the velocity, and the density are the critical pressure, the critical velocity, and the critical density, which are denoted as $P^{*}, V^{*}$, and $\rho^{*}$, respectively:

$$
P^{*}=P_{o}\left(\frac{2}{\kappa+1}\right)^{\frac{\kappa}{\kappa-1}}
$$

Assuming an ideal gas, when the inner pressure of the concentration probe drops below the critical pressure, $P^{*}$, the maximum mass flow rate, $m_{\max }$, through the convergent nozzle is estimated using the continuity equation, as follows:

$$
m_{\max }=A_{s n} V^{*} \rho^{*}=A_{s n} \sqrt{\frac{2 \kappa}{\kappa+1} \frac{P_{o}}{\rho_{o}}} \rho_{o}\left(\frac{P^{*}}{P_{o}}\right)^{1 / \kappa}=P_{o} A_{s n} \sqrt{\frac{\kappa}{R T_{o}}}\left(\frac{2}{\kappa+1}\right)^{\frac{1}{2} \frac{(\kappa+1)}{(\kappa-1)}}
$$

where the atmospheric pressure, $P_{o}$, is $101.3 \times 10^{3} \mathrm{~Pa}$, the sonic nozzle exit area, $A_{s n}$, is $\pi d t^{2} / 4=\pi\left(55 \times 10^{-6}\right)^{2} / 4=2.37 \times 10^{-9} \mathrm{~m}^{2}$, the gas constant, $R$, of air is $287 \mathrm{~J} /(\mathrm{kg} \cdot \mathrm{K}), T_{o}$ is $283 \mathrm{~K}$, the ratio of specific heats, $\kappa$, is 1.4 , and the maximum mass flow rate, $m_{\max }$, is $5.7 \times$ $10^{-7} \mathrm{~kg} / \mathrm{s}$. The volume flow rate under atmospheric pressure is $4.7 \times 10^{-7} \mathrm{~m}^{3} / \mathrm{s}$. For the case in which the issuing nozzle diameter is $8.5 \mathrm{~mm}$, the issuing gas is air, and the issuing Reynolds number, $R e$, is $4.0 \times 10^{3}$, the issuing flow rate becomes $4.0 \times 10^{-4} \mathrm{~m}^{3} / \mathrm{s}$. The suction flow rate of the sensor is thought to be approximately $0.1 \%$ of the jet flow rate. Since the ratio of the sensor suction flow to the jet flow increases due to low velocity around the nozzle, it is necessary to measure concentration carefully near the nozzle exit.

\section{Concentration Distribution of Buoyant Jet Diffusion}

\subsection{Experimental Setup and Flow Visualization}

The experimental setup is shown in Fig. 9. Gas cylinders supplied helium or carbon dioxide. A pure gas flow rate was measured by the orifice flow meter. The issuing nozzle was a converging round nozzle of $8.5 \mathrm{~mm}$ in diameter and a contraction ratio of 0.30 . The exit velocity distribution was a top-hat distribution. The nozzle directions were vertically upward or downward. The concentration and velocity distributions were measured by the concentration sensor and forward scattering LDV using a He-Ne laser light source. 


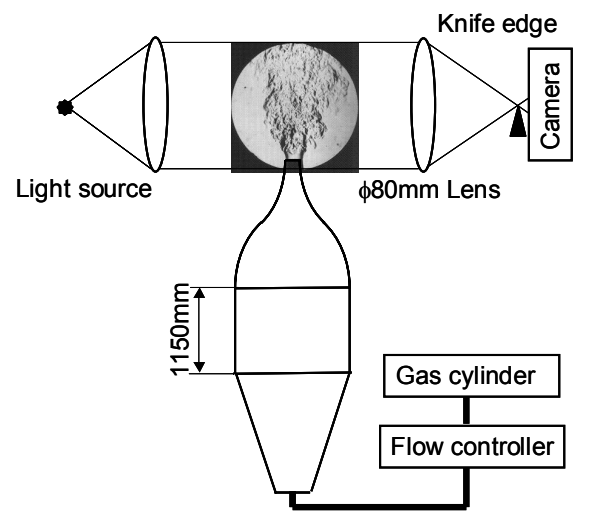

Fig. 9 Experimental setup

In order to clarify the diffusion of the jet, Schlieren visualizations of the vertical upward helium and carbon dioxide jets are shown in Fig. 10. The issuing Reynolds number range was from $R e=1 \times 10^{3}$ to $10 \times 10^{3}$. The Schlieren device consisted of $80 \mathrm{~mm}$ lenses, a knife edge, and a xenon lamp as a light source. In the case of the helium jet, the potential core is very short for $R e=3 \times 10^{3}$ to $8 \times 10^{3}$, and the helium expands chaotically into the surroundings immediately after issue. This expansion is suppressed for $R e=10 \times 10^{3}$, as compared to $R e=5 \times 10^{3}$. In the carbon dioxide jet, a vortex ring having a scale equal to the jet flow diameter is generated at a downstream distance of several times the nozzle diameter at $R e=3 \times 10^{3}$ and $5 \times 10^{3}$. These vortex rings later collapse and diffuse. The scale of the vortex ring decreases to the above-mentioned size. Moreover, the process of vortex ring collapse is not clear at $R e=10 \times 10^{3}$.

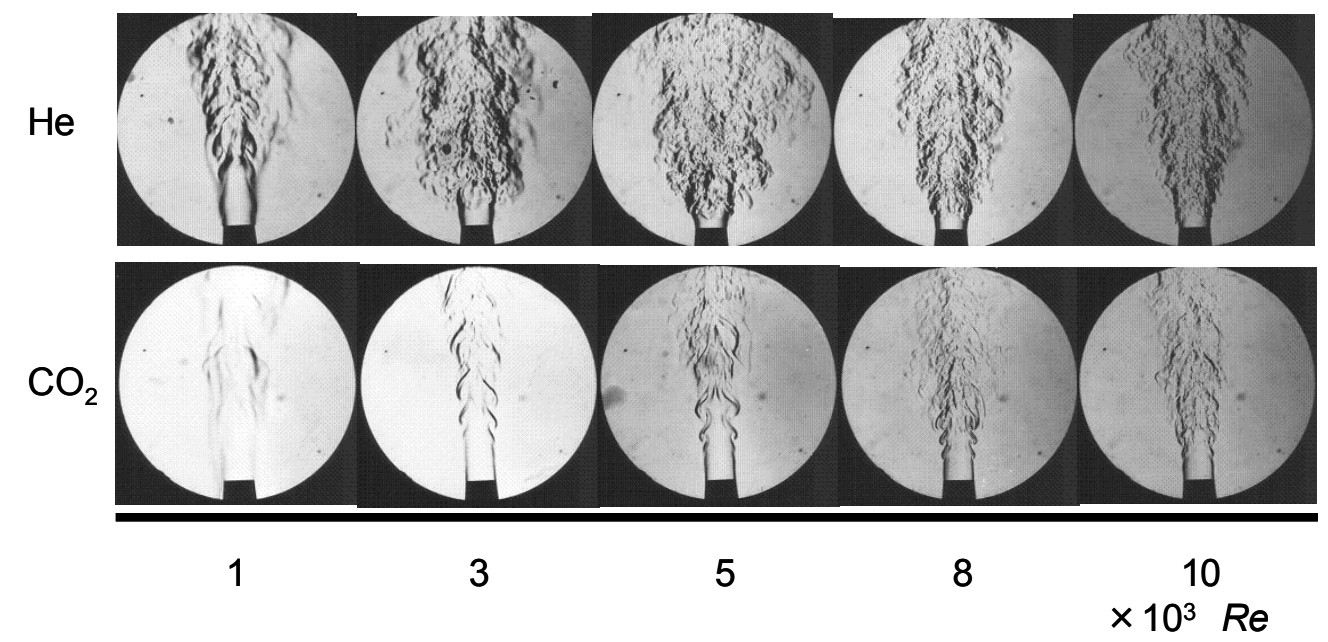

Fig. 10 Schlieren photographs of the $\mathrm{He}$ jet and the $\mathrm{CO}_{2}$ jet

\subsection{Distributions of Velocity and Concentration}

Figure 11 shows contour maps of the velocity and concentration of the helium jet. The issuing Reynolds number is $R e=2.3 \times 10^{3}$. The upward jet is indicated by the dotted lines, and the solid lines indicate the downward jet. The velocity contour map reveals that the velocity decreases to $U / U o=0.3$ at $X / d=9$, for both upward and downward velocities. When we consider the velocity contour line of $U / U o=0.05$, a difference in the velocity contour line is observed between the upward and downward velocities. In the case of the upward jet, the positive buoyancy maintains the velocity. The velocity decreases rapidly because of the negative buoyancy in the downward jet, and the backflow can be observed to be caused by Schlieren photographs at $X / d=40$. This region is thought to be a buoyancy region. Comparison of the concentration contour with the velocity contour reveals the 
following. When you compare the ranges of diffusion to the surroundings with using Fig.11 (a) and (b), it is understood that the concentration contour extends farther than the velocity contour. When we consider the concentration contour line of $\Lambda=0.3$ on the jet axis, the concentration contour line is located at $X / d=25$ for the upward jet and at $X / d=38$ for the downward jet. The jet flow advances because of the positive buoyancy, even if the flow velocity decreases in the upward jet and the concentration decreases in the issuing direction. On the other hand, the downward jet does not diffuse in the traveling direction, and the helium concentration lowers extremely in the region from $X / d=30$ to $X / d=40$, because the jet reaches a maximum distance due to the negative buoyancy, and the flow reverses.

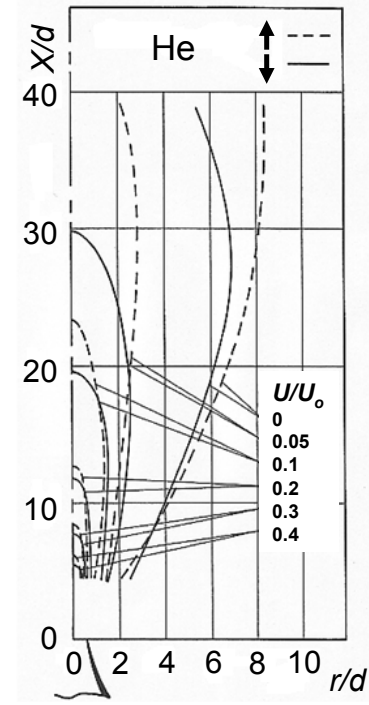

(a) Velocity

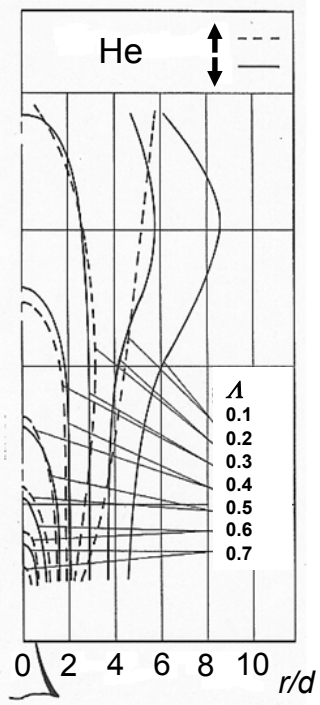

(b) Concentration

Fig.11 Contour of velocity and concentration ( $\mathrm{He}$ jet, $\left.R e=2.3 \times 10^{3}\right)$

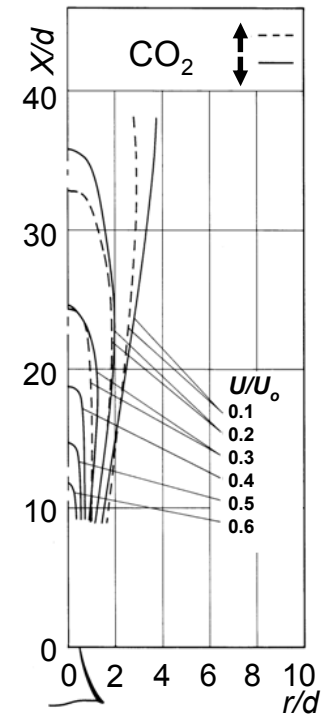

(a) Velocity

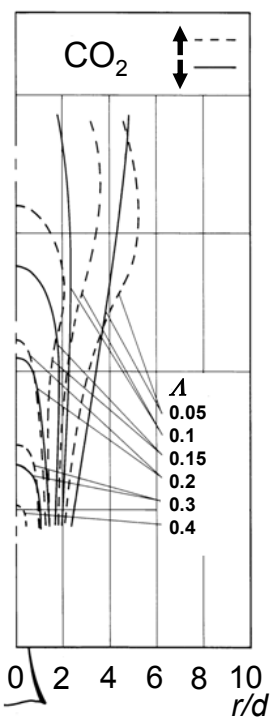

(b) Concentration

Fig. 12 Contour of velocity and concentration $\left(\mathrm{CO}_{2}\right.$ jet, $\left.R e=9.5 \times 10^{3}\right)$

Figure 12 shows the contour maps of velocity and concentration for the carbon dioxide jet. The issuing Reynolds number is $R e=9.5 \times 10^{3}$. The vertical upward jet is indicated by the dotted lines, and the downward jet is indicated by the solid lines. The velocity contour map reveals the following. The velocity decreases to $U / U o=0.3$ at $X / d=25$, for both the upward and downward jets. This distance is 2.5 times longer than that for the helium jet. 
The distance change due to the difference in density of the issuing gas is remarkable. In the case of an upward jet, the jet loses momentum continuously due to the negative buoyancy force and is eventually directed downward. The concentration contour line reveals the following. At $\Lambda=0.15$, the jet flow center is located at $X / d=27$ for the downward jet because of the positive buoyancy and is located at $X / d=32$ for the upward jet affected by negative buoyancy. The carbon dioxide remains and the concentration diffusion in the direction of the jet flow axis weakens when the negative buoyancy acts toward the maximum height position, at which the jet is eventually directed downward. Comparison of the expansion of the velocity and concentration contour lines in the direction of the surroundings reveals only a slight difference. This is different from the case of helium. This is thought to be caused by the increase in the difference between the momentum diffusion and the concentration diffusion because the density of helium with air is larger than the density of carbon dioxide.

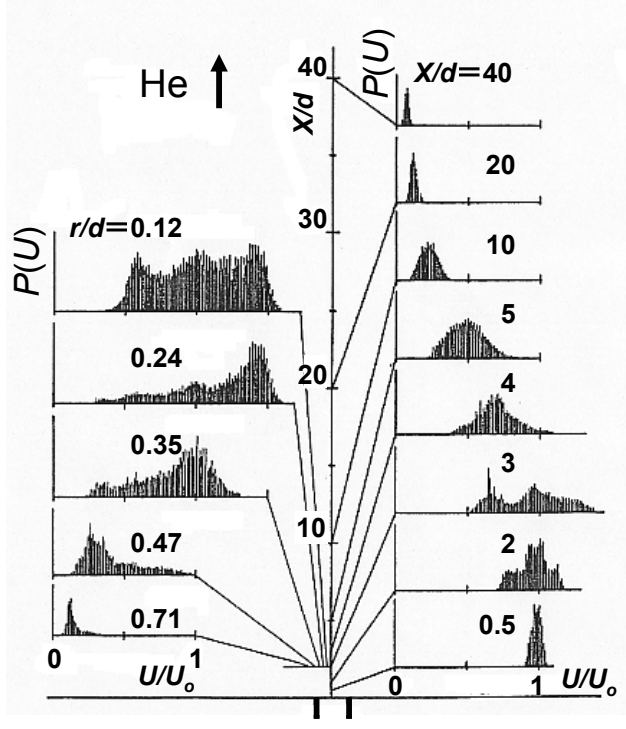

(a) Velocity

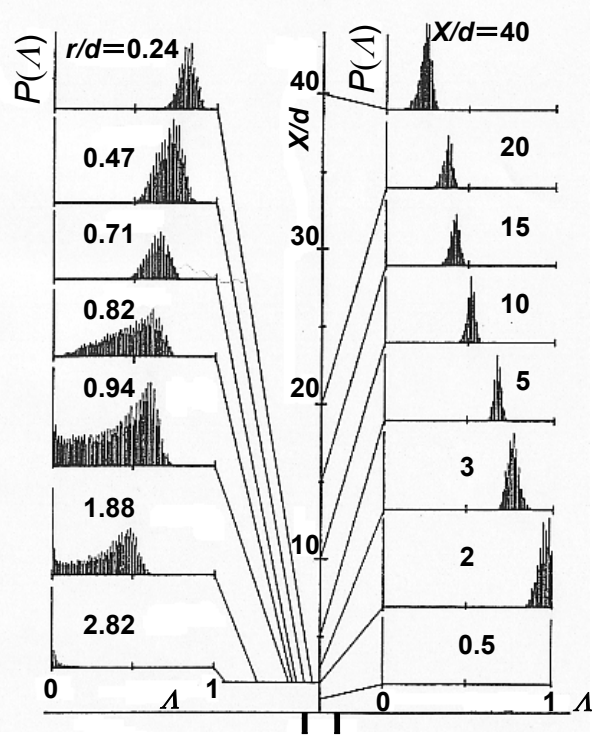

(b) Concentration

Fig.13 Probability density function of velocity and concentration (He jet, $R e=2.8 \times 10^{3}$ )

\subsection{Probability Density Function of Velocity and Concentration of the He Jet}

In order to clarify the diffusion of the helium jet, the probability density functions (PDF) of the velocity data obtained by LDV and the concentration data obtained by the concentration sensor are shown in Fig. 13. The issuing Reynolds number is $\operatorname{Re}=2.8 \times 10^{3}$. The variation of PDF with respect to velocity reveals that the issuing velocity is maintained at $X / d=0.5$ on the jet flow center axis. However, there are three peaks on the PDF of concentration at $X / d=2$. This indicates that the sensor output has sinusoidal wave and fluctuation. This is thought to be caused by the velocity turbulence generated by the passage of the vortex ring generated in the first stage of the jet flow. The PDF of velocity exhibits an almost Gaussian distribution at $X / d=5$. The velocity decreases rapidly in the axial direction from $X / d=10$ to 40 and also in the radial direction from $r / d=0$ to 0.71 .

The PDF of concentration reveals the following. The jet concentration $\Lambda=1$ (at the nozzle exit) is maintained at $X / d=0.5$ on the jet axis. The fluctuating concentration, $\Lambda^{\prime}=$ 0.05 is generated at $X / d=2$. This is the region in which the vortex ring collapses, and active diffusion is assumed to progress from the change in the PDF of velocity. No rapid changes are observed from $X / d=10$ to 40 . The PDF of the concentration shifts to a Gaussian distribution.

On the other hand, in the radial direction at $X / d=2$, PDF distributions of the inside of 
the nozzle section $(r / d<1)$ become Gaussian. PDF distributions of the outside of the nozzle section $(1<r / d<3)$ exhibit a wide variation from $\Lambda=0$ to 0.7 and become approximately $\Lambda=0$ at $r / d=3$. The velocity distribution is thought to be related to the concentration distribution. In the area around $X / d=2$, both the PDF of the velocity and the concentration change remarkably in the vicinity of the center as the vortex ring passes and collapses. Helium $(\Lambda=0.5)$ is believed to remain around the potential core of the jet due to the momentum loss.

\section{Conclusion}

A concentration sensor using a hot wire and a sonic nozzle has been applied to the measurement of the concentration of jet diffusion. The static and dynamic characteristics of the sensor are calibrated. Measurement of the concentration distribution and the velocity distribution of the buoyant jets flow revealed the following.

The time constant of the sensor, $\tau$ is $0.74 \mathrm{~ms}$ for helium and $3.48 \mathrm{~ms}$ for carbon dioxide, if the sonic nozzle diameter is $55 \mu \mathrm{m}$, the pressure ratio inside to outside of the sensor is 0.02 , and the gap between the sonic nozzle exit and the hot wire is $0.65 \mathrm{~mm}$.

Using the concentration sensor and LDV, the velocity and concentration contours of the helium and carbon dioxide jets were obtained. When an negative buoyancy occurs, a high-density area remains because a maximum distance due to the negative buoyancy exists. The concentration diffuses to the radial direction more widely than the momentum. In addition, the effect is more remarkable for He gas due to the large difference in density between air and He.

\section{References}

(1) Grinstein, F. F., Vortex dynamics and entrainment in rectangular free jets, Journal of Fluid Mechanics, Vol. 437 (2001), pp. 69-101.

(2) Miyagi, N., Hodoya, H., Fujita, H., Shoji, H., Kimura, M., Study of Active Jet Control by Acoustically Driven Secondary Film Flow Influence of Velocity Ratio and Acoustic Strouhal Number, The Japan Society of Mechanical Engineers International Journal, Series B, Vol. 49, No. 4 (2006), pp. 974-979.

(3) Shakouchi, T., Durst, F., Toyoda, K., edited, Jets Wakes and Separated Flows, (2005), The Japan Society of Mechanical Engineers No. 05-201.

(4) Kurimoto, N., Suzuki, Y., Kasagi, N., Active Control Coaxial Jet Mixing and Combustion with Arrayed Micro Actuators, Proceedings of Experimental Heat Transfer, Fluid Mechanics, and Thermodynamics 2001, Vol. 1 (2001), pp. 511-516.

(5) Takahashi, K. and Sasaki, A, Advanced Sensor Handbook (in Japanese), (1994), pp.174-183, pp.209-216, pp.700-701, Baifukan Co., Ltd.

(6) Brown, G. L., Rebollo, M. R., A Small Fast-Response Probe to Measure Composition of a Binary Gas Mixture, AIAA Journal, Vol. 10, No. 5 (1972), pp. 649-652.

(7) Era, Y., A Method of Concentration Measurement in Gas Flow, Report of the Research Institute of Science and Technology, Nihon University, Vol. 21 (1977), pp. 9-26.

(8) Muramatsu, A., Era, Y., Measurements of Concentration and Velocity in a Gas Mixture (A Method Utilizing a Piezoresistive Sensor and a Hot-Wire Concentration Probe) Transactions of the Japan Society of Mechanical Engineers, Series B (in Japanese), Vol. 64, No. 624 (1998), pp. 2632-2639. 